\title{
Revisiting anti-angiogenic therapy for recurrent glioblastoma
}

\author{
Katherine B. Peters \\ The Preston Robert Tisch Brain Tumor Center, Duke University, Durham, NC, USA \\ Correspondence to: Katherine B. Peters, MD, PhD, FAAN. The Preston Robert Tisch Brain Tumor Center, Duke University Medical Center, PO Box \\ 3624, Durham, NC 27710, USA. Email: katherine.peters@duke.edu. \\ Comment on: Lombardi G, De Salvo GL, Brandes AA, et al. Regorafenib compared with lomustine in patients with relapsed glioblastoma (REGOMA): \\ a multicentre, open-label, randomised, controlled, phase 2 trial. Lancet Oncol 2019;20:110-9.
}

Submitted May 01, 2019. Accepted for publication May 20, 2019.

doi: $10.21037 /$ tcr.2019.05.25

View this article at: http://dx.doi.org/10.21037/tcr.2019.05.25

Glioblastoma (GBM) represents the most common malignant primary brain neoplasm in adult patients with a median overall survival (OS) in a modern US population-based cancer registry data Surveillance, Epidemiology, and End Results (SEER) of 11 and 14.6 months based on standard of care therapy $(1,2)$. Despite advances in treatment, GBM (WHO grade IV) is associated with considerable morbidity and mortality as compared with other malignancies.

Recurrent disease is nearly inevitable for all patients diagnosed with this cancer. Once a GBM patient experiences relapse and/or recurrence, they have a poor median OS with modern phase 3 study with OS ranging from 8 to 9 months even with utilization of FDA approved standard of care treatment regimens (3). Bevacizumab, antivascular endothelial growth factor-A (VEGF-A) antibody, was initially promising given the highly vascularized nature of GBM and the phase 2 studies by Taal et al. and Friedman et al. demonstrated favorable results $(4,5)$. In particular, the BELOB study, a phase 2 study designed to compare bevacizumab alone versus lomustine alone and lomustine in combination with bevacizumab, showed an improved 9-month OS survival in the combination group receiving bevacizumab with lomustine in comparison to lomustine alone (59\% versus $38 \%$, respectively) (5). These results prompted the phase 3 EORTC trial randomizing patients to either lomustine alone or bevacizumab in combination with lomustine (3). Despite the initial phase 2 findings that the combination improved outcomes, this phase 3 study failed to show superiority of the combination in comparison to lomustine monotherapy with median OS of 9.1 months (95\% CI, 8.1-10.1 months) in the combination group and 8.6 months (95\% CI, 7.6-10.4 months) in the monotherapy group (3). Neuro-oncologists have sought the appropriate anti-angiogenic agent or combination of anti-angiogenic and chemotherapy for the treatment of recurrent GBM, but phase 3 studies have remained unable to improve OS outcomes (6). This holds true for phase 3 studies in newly diagnosed GBM patients as the addition of bevacizumab to standard of care concurrent radiation therapy with temozolomide followed by adjuvant temozolomide failed to extend median OS (79). Beyond bevacizumab, investigators have studied antiangiogenic in phase 3 trials including cilengitide, intravenous $\alpha_{\mathrm{v}}$ integrin inhibitor, and cediranib, an orally available panVEGF receptor tyrosine kinase inhibitor, but remarkable results have failed to be achieved for these studies in recurrent and newly diagnosed GBM (10-12). Theories point to the fact that while VEGF receptor is responsible for angiogenic growth in GBM that there are other signaling pathways that can be co-opted to promote growth that are complementary or agnostic to angiogenesis (13).

The REGOMA trial published by Lombardi and colleagues utilizes regorafenib, an agent that targets signaling pathways involved in angiogenesis and other growth pathways thereby confronting the challenges that bevacizumab faced in the treatment of GBM (14). Regorafenib is an orally available multi-kinase inhibitor that targets angiogenic, stromal and oncogenic receptor tyrosine kinases $(15,16)$. Tyrosine receptor kinases targeted include fibroblast growth factor receptor (FGFR), platelet derived growth factor receptor- $\beta$ (PDGFR- $\beta$ ), VEGF receptor, tyrosine kinase with immunoglobulin and epidermal growth factor homology domain 2 (TIE-2), and proto-oncogenic tyrosine kinase receptors c-KIT and RET. Regorafenib was first FDA approved in 2012 for use in metastatic colorectal cancer and has subsequently received approval in advanced gastrointestinal stromal tumors and advanced hepatocellular 
carcinoma (17-20). Common toxicities of regorafenib are hand-foot-skin reactions, voice changes, mucositis, diarrhea, and hypertension and reflect the multikinase activity of this potential therapeutic agent. Notably, phase 1 trial of regorafenib in combination with cetuximab, intravenous monoclonal antibody of epidermal growth factor receptor (EGFR), in subjects with advanced cancers included a subject with relapsed GBM, and the investigators observed a clinical response in this relapsed GBM subject (21). The multimodal activity of regorafenib, its positive antitumor activity in GBM xenograft models, and this singular experience with regorafenib in a patient with GBM prompted investigators to explore the use of regorafenib in relapsed GBM patients $(22,23)$.

Investigators of the REGOMA trial recently published their results of their multicenter, open-label, randomized, controlled, phase 2 trial (14). In this trial, 119 GBM patients after their first confirmed progression after standard of care therapy (best possible surgery followed by concurrent radiation therapy and temozolomide) were randomized in a 1:1 fashion to control group (lomustine $110 \mathrm{mg} / \mathrm{m}^{2}$ orally on day 1 of every 6 -week cycle) or to the experimental group (regorafenib $160 \mathrm{mg}$ orally once daily for first 3 weeks of every 4-week cycle). Investigators performed regular radiographic assessments every 8 weeks to evaluate for tumor response by RANO criteria. Primary endpoint of the study was OS. The cohorts were 59 patients in the experimental group and 60 patients in the control group with relatively similar distributions in sex, ECOG performance status, and isocitrate dehydrogenase (IDH) mutation status. There was improvement in the median OS of subjects in the regorafenib group [7.4 months (95\% CI, 5.8-12.0 months)] in comparison to the control group that received lomustine monotherapy [5.6 months (95\% CI, 4.7-7.3 months)]. Importantly, the hazard ratio was 0.50 (95\% CI, 0.33-0.74) with a significant difference remarkable for increased survival in the regorafenib group $(\mathrm{P}=0.0009)$. Investigators documented similar adverse events for regorafenib group in this phase 2 trial with fatigue, hand-foot-skin reaction, hypertension, and diarrhea being examples of these observations. Therefore, based on multicenter, open-label, randomized, controlled phase 2 trial, there is renewed hope for new therapeutics utilizing anti-angiogenics in relapsed GBM.

For the neuro-oncology community, a successful phase 2 clinical trial in relapsed GBM is often met with a balance of optimism and skepticism. Experiences of promising therapeutic agents from phase 2 clinical trials such as the BELOB trial comparing bevacizumab monotherapy, lomustine monotherapy, and combination of bevacizumab with lomustine and subsequent lack of congruence with a similar phase 3 randomized controlled trial leads neurooncologists to take pause at successful phase 2 studies. Particular to REGOMA, while some patient characteristics were balanced such as ECOG performance status and IDH mutation status, there was a trend towards more favorable patient characteristics in the regorafenib group. These were younger age, higher percentage of methylated MGMT promoter status, longer median time to relapse from initial surgery and/or radiation therapy, and lower corticosteroid usage at time of enrollment. This puts into question whether the regorafenib group has more favorable prognostic factors in comparison to control group.

This apparent imbalance between the control and experimental groups is also highlighted in regards to treatment regimens after progression on this study. A higher percentage of patients in the regorafenib group received third-line therapy than patients in the lomustine group. One rationale for this observation is that more favorable prognostic factors evidenced in the regorafenib group at the time of enrollment allowed them to move forward with more therapeutics even in the third-line setting.

The obvious comparator to this phase 2 study is the BELOB study that this editorial continues to reference (5). Median OS of lomustine at a similar dose of $110 \mathrm{mg} / \mathrm{m}^{2}$ in the BELOB study was 8 months (95\% CI, 6-11 months) and in the following phase 3 study comparing single agent lomustine to combination lomustine and bevacizumab, the median OS in the lomustine monotherapy $\left(110 \mathrm{mg} / \mathrm{m}^{2}\right)$ was 8.6 months (95\% CI, 7.6-10.4 months) (3). It is important to note that this median OS for lomustine monotherapy exhibited in the phase 3 study of lomustine monotherapy in comparison to lomustine and bevacizumab combination therapy is also superior to the lomustine monotherapy arm in REGOMA. The striking inferiority of lomustine monotherapy in the REGOMA study provides reflection of whether a larger randomized phase 3 study can aid in understanding whether the signal towards improvement is real for the regorafenib treated group (Table 1). Other studies in recurrent GBM have shown similar median OS for lomustine monotherapy. The phase 3 trial of enzastaurin, an oral serine/threonine kinase inhibitor, in comparison to control group of lomustine (100 to $130 \mathrm{mg} / \mathrm{m}^{2}$ on day 1 in 6-week cycle) demonstrated a median OS [7.1 months (95\% CI, 6.0-8.8 months)] more comparable to BELOB and this was conducted before widespread use of bevacizumab as a salvage treatment (24). The phase 3 trial of cediranib in comparison to combination cediranib with lomustine and 
Table 1 Comparison of phase 2 and 3 recurrent GBM trials with lomustine monotherapy control groups

\begin{tabular}{|c|c|c|c|c|c|}
\hline $\begin{array}{l}\text { Experimental agent and/or } \\
\text { combination (study) }\end{array}$ & Enzastaurin (24) & $\begin{array}{l}\text { Cediranib* or cediranib } \\
\quad+\text { lomustine }(12)\end{array}$ & $\begin{array}{c}\text { Bevacizumab** or } \\
\text { bevacizumab + } \\
\text { lomustine (5) }\end{array}$ & $\begin{array}{l}\text { Bevacizumab + } \\
\text { lomustine (3) }\end{array}$ & Regorafenib (14) \\
\hline Randomization & $2: 1$ & $2: 2: 1$ & $1: 1: 1$ & $2: 1$ & $1: 1$ \\
\hline \multicolumn{6}{|l|}{ Number } \\
\hline Experimental group & 174 & 131 & 50 & 288 & 59 \\
\hline \multicolumn{6}{|c|}{ Median overall survival (95\% Cl) (months) } \\
\hline Lomustine monotherapy group & $7.1(6.0-8.8)$ & 9.8 & $8[6-11]$ & $8.6(7.6-10.4)$ & $5.6(4.7-7.3)$ \\
\hline Experimental group & $6.6(5.2-7.8)$ & 8.0 & $8[6-9]$ & $9.1(8.1-10.1)$ & $7.4(5.8-12.0)$ \\
\hline
\end{tabular}

*, only reporting cediranib monotherapy group in this table; ${ }^{\star \star}$, only reporting bevacizumab monotherapy group in this table. n/a, not available.

lomustine monotherapy further documented a median OS of 9.8 months in the lomustine monotherapy group (12). Based on this historical data with lomustine monotherapy in recurrent GBM, one must question the obvious inferiority of lomustine monotherapy in the REGOMA study.

Given the obvious and apparent toxicities of regorafenib with skin reactions such as hand-foot-skin syndrome, it will be challenging to proceed with a blinded study. Moving forward, it will benefit investigators in the phase 3 of regorafenib to power the study appropriately so that a true understanding of the control/comparator group can be demonstrated. The authors acknowledge these shortcomings in this study and concede that moving forward the study needs to have more balanced patient characteristics, increased power, and independent centralized review of magnetic resonance imaging (MRI) imaging in the assessment of progression.

In conclusion, results from the study by Lombardi and colleagues are promising and give hope to the use of antiangiogenics in relapsed GBM. Given the side effect profile of this targeted agent, investigators should choose the appropriate health-related quality of life patient-reported outcomes instruments to capture the patient and caregiver experience with an agent such as regorafenib. Lombardi and colleagues conclude in their manuscript that the results of REGOMA warrants investigation of regorafenib in a larger phase 3 clinical trial. While this is the logical next step for the development of regorafenib as a potential therapeutic in recurrent GBM population, it is important to advise the potential investigators of a future phase 3 trial for regorafenib in recurrent GBM to design carefully the power of the study, to understand the balance of patient characteristics between the control and experimental groups, and to select appropriately the control therapeutic agent.

\section{Acknowledgments}

Funding: None.

\section{Footnote}

Provenance and Peer Review: This article is commissioned and reviewed by the Section Editor Xian-Xin Qiu, MD, $\mathrm{PhD}$ candidate [Shanghai Proton and Heavy Ion Center (SPHIC), a.k.a. the Proton and Heavy Ion Center of Fudan University Shanghai Cancer Center (FUSCC), Shanghai, China].

Conflicts of Interest: The author has completed the ICMJE uniform disclosure form (available at http://dx.doi. org/10.21037/tcr.2019.05.25). The author has no conflicts of interest to declare.

Ethical Statement: The author is accountable for all aspects of the work in ensuring that questions related to the accuracy or integrity of any part of the work are appropriately investigated and resolved.

Open Access Statement: This is an Open Access article distributed in accordance with the Creative Commons Attribution-NonCommercial-NoDerivs 4.0 International License (CC BY-NC-ND 4.0), which permits the noncommercial replication and distribution of the article with the strict proviso that no changes or edits are made and the original work is properly cited (including links to both the formal publication through the relevant DOI and the license). See: https://creativecommons.org/licenses/by-nc-nd/4.0/. 


\section{References}

1. Johnson DR, Omuro AMP, Ravelo A, et al. Overall survival in patients with glioblastoma before and after bevacizumab approval. Curr Med Res Opin 2018;34:813-20.

2. Stupp R, Mason WP, van den Bent MJ, et al. Radiotherapy plus concomitant and adjuvant temozolomide for glioblastoma. N Engl J Med 2005;352:987-96.

3. Wick W, Gorlia T, Bendszus M, et al. Lomustine and Bevacizumab in Progressive Glioblastoma. N Engl J Med 2017;377:1954-63.

4. Friedman HS, Prados MD, Wen PY, et al. Bevacizumab alone and in combination with irinotecan in recurrent glioblastoma. J Clin Oncol 2009;27:4733-40.

5. Taal W, Oosterkamp HM, Walenkamp AM, et al. Singleagent bevacizumab or lomustine versus a combination of bevacizumab plus lomustine in patients with recurrent glioblastoma (BELOB trial): a randomised controlled phase 2 trial. Lancet Oncol 2014;15:943-53.

6. Ghiaseddin A, Peters KB. Use of bevacizumab in recurrent glioblastoma. CNS Oncol 2015;4:157-69.

7. Gilbert MR, Sulman EP, Mehta MP. Bevacizumab for newly diagnosed glioblastoma. N Engl J Med 2014;370:2048-9.

8. Gilbert MR, Dignam JJ, Armstrong TS, et al. A randomized trial of bevacizumab for newly diagnosed glioblastoma. N Engl J Med 2014;370:699-708.

9. Chinot OL, de La Motte Rouge T, Moore N, et al. AVAglio: Phase 3 trial of bevacizumab plus temozolomide and radiotherapy in newly diagnosed glioblastoma multiforme. Adv Ther 2011;28:334-40.

10. Nabors LB, Fink KL, Mikkelsen T, et al. Two cilengitide regimens in combination with standard treatment for patients with newly diagnosed glioblastoma and unmethylated MGMT gene promoter: results of the open-label, controlled, randomized phase II CORE study. Neuro Oncol 2015;17:708-17.

11. Stupp R, Hegi ME, Gorlia T, et al. Cilengitide combined with standard treatment for patients with newly diagnosed glioblastoma with methylated MGMT promoter (CENTRIC EORTC 26071-22072 study): a multicentre, randomised, open-label, phase 3 trial. Lancet Oncol 2014;15:1100-8.

12. Batchelor TT, Mulholland P, Neyns B, et al. Phase III randomized trial comparing the efficacy of cediranib as monotherapy, and in combination with lomustine, versus lomustine alone in patients with recurrent glioblastoma. J Clin Oncol 2013;31:3212-8.

13. Zhao Y, Adjei AA. Targeting Angiogenesis in Cancer Therapy: Moving Beyond Vascular Endothelial Growth Factor. Oncologist 2015;20:660-73.
14. Lombardi G, De Salvo GL, Brandes AA, et al. Regorafenib compared with lomustine in patients with relapsed glioblastoma (REGOMA): a multicentre, open-label, randomised, controlled, phase 2 trial. Lancet Oncol 2019;20:110-9.

15. Schmieder R, Hoffmann J, Becker M, et al. Regorafenib (BAY 73-4506): antitumor and antimetastatic activities in preclinical models of colorectal cancer. Int J Cancer 2014;135:1487-96.

16. Wilhelm SM, Dumas J, Adnane L, et al. Regorafenib (BAY 73-4506): a new oral multikinase inhibitor of angiogenic, stromal and oncogenic receptor tyrosine kinases with potent preclinical antitumor activity. Int J Cancer 2011;129:245-55.

17. Yoshino T, Komatsu Y, Yamada Y, et al. Randomized phase III trial of regorafenib in metastatic colorectal cancer: analysis of the CORRECT Japanese and non-Japanese subpopulations. Invest New Drugs 2015;33:740-50.

18. Grothey A, Van Cutsem E, Sobrero A, et al. Regorafenib monotherapy for previously treated metastatic colorectal cancer (CORRECT): an international, multicentre, randomised, placebo-controlled, phase 3 trial. Lancet 2013;381:303-12.

19. Demetri GD, Reichardt P, Kang YK, et al. Efficacy and safety of regorafenib for advanced gastrointestinal stromal tumours after failure of imatinib and sunitinib (GRID): an international, multicentre, randomised, placebocontrolled, phase 3 trial. Lancet 2013;381:295-302.

20. Bruix J, Qin S, Merle P, et al. Regorafenib for patients with hepatocellular carcinoma who progressed on sorafenib treatment (RESORCE): a randomised, double-blind, placebo-controlled, phase 3 trial. Lancet 2017;389:56-66.

21. Subbiah V, Khawaja MR, Hong DS, et al. First-in-human trial of multikinase VEGF inhibitor regorafenib and antiEGFR antibody cetuximab in advanced cancer patients. JCI Insight 2017. doi: 10.1172/jci.insight.90380.

22. Hamed HA, Tavallai S, Grant S, et al. Sorafenib/ regorafenib and lapatinib interact to kill CNS tumor cells. J Cell Physiol 2015;230:131-9.

23. Sajithlal GB, Hamed HA, Cruickshanks N, et al. Sorafenib/regorafenib and phosphatidyl inositol 3 kinase/ thymoma viral proto-oncogene inhibition interact to kill tumor cells. Mol Pharmacol 2013;84:562-71.

24. Wick W, Puduvalli VK, Chamberlain MC, et al. Phase III study of enzastaurin compared with lomustine in the treatment of recurrent intracranial glioblastoma. J Clin Oncol 2010;28:1168-74.

Cite this article as: Peters KB. Revisiting anti-angiogenic therapy for recurrent glioblastoma. Transl Cancer Res 2019;8(Suppl 6):S569-S572. doi: 10.21037/tcr.2019.05.25 